\section{Automated retinal shadow compensation of optical coherence tomography images}

\author{
Tapio Fabritius, ${ }^{\mathrm{a}, \mathrm{b}}$, Shuichi Makita, ${ }^{\mathrm{a}}$ Yongjoo Hong, ${ }^{\mathrm{c}}$ \\ Risto Myllylä, ${ }^{b}$ and Yoshiaki Yasuno ${ }^{a}$ \\ aUniversity of Tsukuba, Computational Optics Group, \\ 1-1-1 Tennodai, Tsukuba, Ibaraki 305-8573 Japan \\ ${ }^{\mathrm{b}}$ University of Oulu, Optoelectronics and Measurement \\ Techniques Laboratory, Erkki Koiso-Kanttilan katu, Oulu, \\ 90014, Finland \\ E-mail: tapio.fabritius@ee.oul.fi \\ 'Korean Advanced Institute of Science and Technology, \\ Department of Mechanical Engineering, 373-1, Guseong- \\ Dong, Yuseong-Gu, Daejeon, 305-701, Korea
}

\begin{abstract}
We present an automated numerical method of compensating for retinal shadows in the choroid. In this method, signal extinction caused by retinal vessels is estimated by subtracting median A-scans obtained from beneath the retinal vessels and A-scans from the surrounding area. Adding the obtained offset vector to A-scans from beneath the retinal vessels allows compensating for shadows in the choroid. In vivo imaging of the human eye was performed by 840-nm-band standard resolution spectral domain optical coherence tomography (SD-OCT), and choroidal vasculature projection images were calculated. Removal of retinal shadows distinctly improved the readability of choroidal images. ๑ 2009 Society of Photo-Optical Instrumentation Engineers. [DOI: 10.1117/1.3076204]
\end{abstract}

Keywords: optical coherence tomography (OCT); image processing; ophthalmology.

Paper 08217LRRR received Jul. 8, 2008; revised manuscript received Nov. 17, 2008; accepted for publication Nov. 26, 2008; published online Feb. 12, 2009.

Ocular vasculature examination is an important way of diagnosing such ophthalmic diseases as age-related macular degeneration (ARMD) and diabetic retinopathy. At this moment, fluorescein angiography (FA) and indocyanine green angiography (ICGA) are frequently used for that purpose. However, both FA and ICGA are limited to 2-D imaging, which sometimes makes it difficult to resolve abnormalities in blood vessels, especially in the choroid. In addition, these methods are rather uncomfortable for the patient due to the mandatory dye injection.

Alternative angiographic methods, based on optical coherence tomography (OCT), have been developed recently to overcome these disadvantages. One of these, presented by Makita et al., is referred to as Doppler optical coherence angiography (DOCA). ${ }^{1}$ This method obtains 3 -D images of the ocular vascular structure by observing Doppler signals caused by blood flow within vessels. ${ }^{1}$ Unfortunately, DOCA requires dense scanning between A-scans, which increases the measurement time. That is problematic, because patients often find it difficult to endure a long measurement time when keep-

FAX: 3588553 2774; E-mail: tapio.fabritius@ee.oulu.fi ing their eyes in a fixed position, causing distortion of 3-D cube data. Moreover, due to the weakness of the Doppler signal obtained from the choroid, the resultant choroidal vasculature images are not very accurate.

Another method, presented by Yasuno et al. and Hong et al. and known as the scattering optical coherence angiography method (SOCA), uses light absorption and the scattering properties of blood to determine retinal and choroidal vessel structures. ${ }^{2,3}$ The significant advantage of SOCA is that, contrary to DOCA, it does not require dense scanning between A-scans, which allows a substantially reduced measurement time. In the SOCA method, the segmentation of vessels is based on the intensity characteristics of OCT signals. However, due to the high absorption of retinal vessels and fringe washout, OCT signals obtained from locations beneath these vessels are significantly decreased. That produces artifacts in the OCT images, known as retinal shadows, thereby decreasing the informativity and readability of vascular images obtained by SOCA. Consequently, there is a real need for an effective method of compensating for the artifacts caused by retinal vessels.

In this paper, we present a numerical method to compensate the artifacts in OCT images caused by retinal vessels.

We used a standard spectral domain optical coherence tomography (SD-OCT) system to obtain 3-D retinal and choroidal OCT images. Since a more detailed description of the measurement system can be found in Ref. 1, this paper will present only a short summary. As a light source, the system used a superluminescent diode (SLD) with a center wavelength of $840 \mathrm{~nm}$ and a FWHM spectral bandwidth of $50 \mathrm{~nm}$. The optical power of the beam on the cornea was $700 \mu \mathrm{W}$, which is less than the ANSI exposure limit. An optical isolator was used to prevent reincident light from entering the source. Polarization controllers were used after the SLD and before the reference and sample arms. A transparent diffractive grating of 1200 lines/mm was used to separate the wavelengths of the coherent light in the spectrometer. The scanning rate of the CCD (Basler, L103k-2k) camera was $18.7 \mathrm{kHz}$, and the exposure time of each A-line was $53.3 \mu \mathrm{s}$. The measured sensitivity of this system was $99.3 \mathrm{~dB}$ at a depth of $100 \mu \mathrm{m}$, while the measured axial resolution in air was $8.8 \mu \mathrm{m}$.

The whole data processing will be briefly described here, with a detailed description of retinal shadow compensation. First, the retina and choroid are separated by identifying the retinal pigment epithelium (RPE) layer from the measured 3-D OCT data (see Fig. 1, step i) When the segmented choroid is flattened to the RPE (step ii), the OCT signal decay at a single depth becomes nearly constant. This enables the use of intensity thresholding-based binarization (ITB) to segment choroidal vessels. A detailed description of this process can be found in an article published by the authors. ${ }^{2,3}$

A new retinal shadow compensation method is used before the segmentation of choroidal vessels. To identify the position and width of retinal shadows, a projection image of retinal vessels is calculated by adding together the obtained logarithmic scale en face slices from the RPE complex (step iii) and binarizing the resultant linear scale image using automatic ITB (step iv). The achieved retinal vessel mask is then used to identify the location of all A-scans of OCT volume that are

1083-3668/2009/14(1)/010503/3/\$25.00 @ 2009 SPIE 


\section{JBO LETTERS}

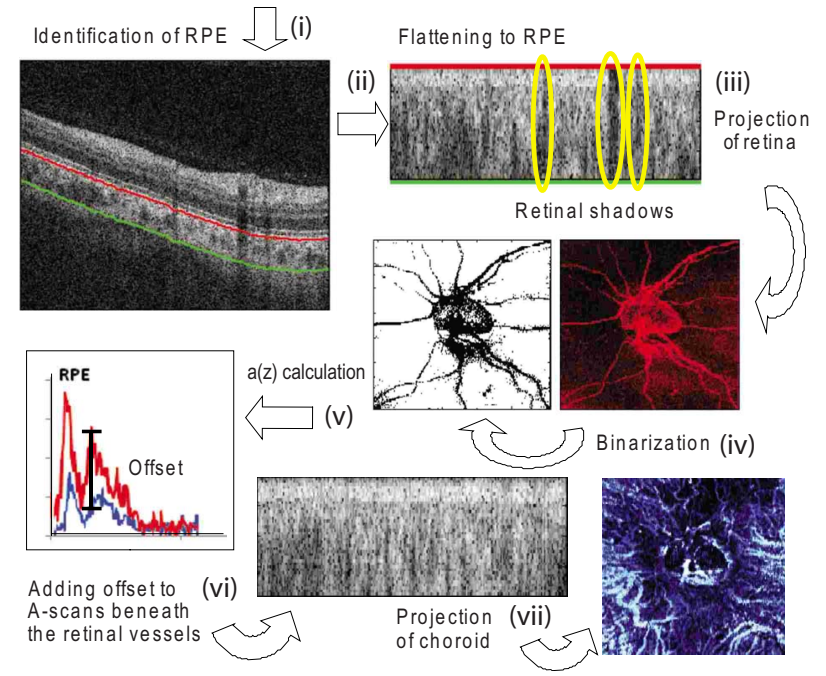

Fig. 1 The steps [(i) to (vii)] of the retinal shadow compensation procedure.

affected by retinal vessels. ${ }^{2,3}$ Logarithmic scaled A-scans with $\left\{I_{j}(z), j \in[1, N]\right\}$ and without $\left\{I_{i}(z), i \in[1, M]\right\}$ the effect of extinction are separated from each other by using the retinal vessel mask. $I_{j}(z)$ and $I_{i}(z)$ are the intensities of OCT signal as a function of depth from RPE $z . N$ and $M$ are the numbers of A-scans with and without the effect of retinal vessels, respectively, and the sum $M+N$ is the number of A-scans of the whole OCT volume. Median A-scans $\widetilde{I}_{i \in[1, M]}(z)$ and $\tilde{I}_{j \in[1, N]}(z)$ are determined by calculating the median intensity of all depths for $I_{j}(z)$ and $I_{i}(z)$, respectively. The function $a(z)$ that estimates signal extinction caused by retinal vessels is obtained by subtracting median A-scans, $a(z)=\widetilde{I}_{i \in[1, M]}(z)$ $-\widetilde{I}_{j \in[1, N]}(z)$. Adding the obtained offset vector to all A-scans beneath the retinal vessels $I_{j}(z)+a(z)$ allows the removal of retinal shadows in thesss choroid (step vi).

After retinal shadow compensation follows the segmentation of choroidal vessels. A vessel segmentation algorithm operates on each en face slice of the OCT volume at constant depths from the RPE. Automatic ITB is performed on each
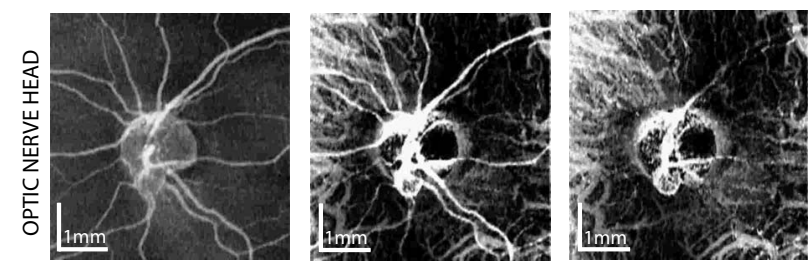

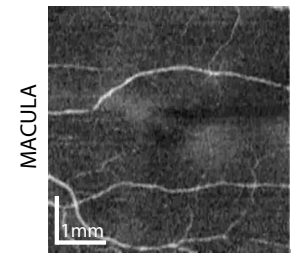

(a)

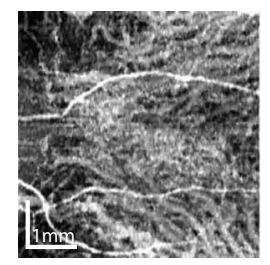

(b)

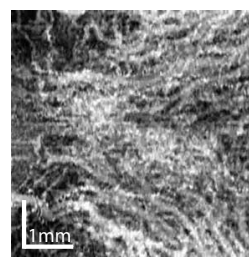

(c)
Fig. 2 SOCA images from optic nerve head $(\mathrm{ONH})$ and macula; (a) projection image of retinal vessels, (b) choroidal vessel without retinal shadow compensation, and (c) choroidal vessels with compensation.

slice containing choroid, producing a 3-D mask of segmented choroidal vessels. Multiplying this mask with intensityinverted OCT volume data allows us to acquire a highcontrast 3-D volume of choroidal vessels. En face projection images of choroidal vessels are obtained by adding all slices from the choroid together (step vii).

To test the method, a 3-D in vivo measurement was performed on a healthy human eye. The optic nerve head $(\mathrm{ONH})$ and macula areas were imaged using $1024 \mathrm{~A}$-scans per frame, with a total of 138 frames. Both $\mathrm{ONH}$ and macula measurement data covered an area of about $5 \mathrm{~mm} \times 5 \mathrm{~mm}$. The required measurement time was $7.7 \mathrm{~s}$. An en face projection image of retinal vessels, shown in Fig. 2(a), was calculated from the RPE. Fig. 2(b) shows the choroidal en face projection image without retinal shadow compensation, while Fig. 2(c) presents the corresponding image with shadow compensation. As seen, the application of the shadow compensation method improves the visibility of choroidal vessels. Some choroidal vessel structures, located beneath the retinal vessels, can be seen in Fig. 2(c).

One advantage of SOCA is the depth-resolved visualization of vessels. To illustrate this feature, Fig. 3 shows en face (a)

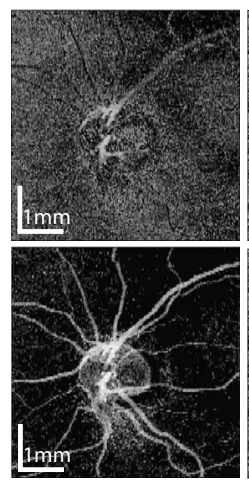

$0 \mu \mathrm{m}$

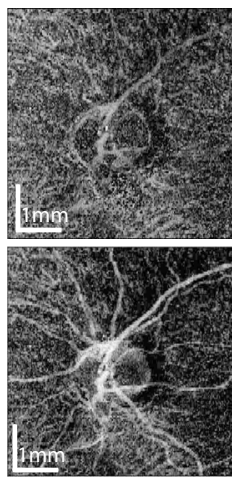

$49 \mu \mathrm{m}$

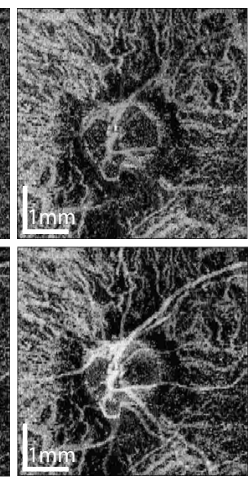

$98 \mu \mathrm{m}$

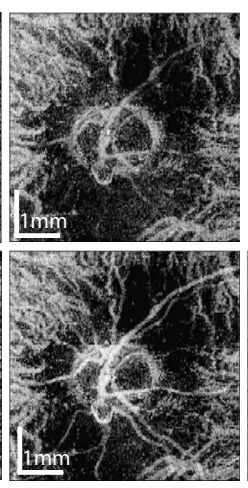

$147 \mu \mathrm{m}$

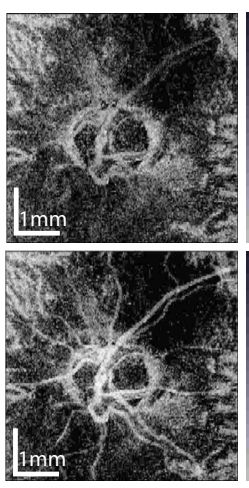

$196 \mu \mathrm{m}$

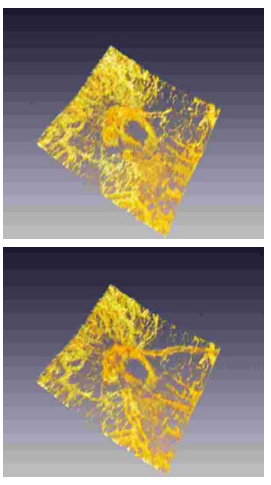

3D volume

Fig. 3 En face slice images of $\mathrm{ONH}$ area. The images in row (a) are obtained as retinal shadow compensation is used, and the images in row (b) without compensation. The first image in both sequences is from the RPE, and the following images are sliced every $49 \mu \mathrm{m}$ relative to the RPE. The last image in both sequences is a 3-D volume rendering of corresponding data. 


\section{JBO LETTERS}

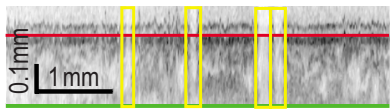

(a)

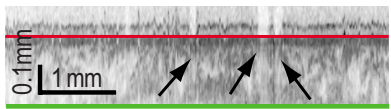

(b)
Fig. 4 Flattened B-scan images with inverted intensity. Image (a) is without and image (b) is with retinal shadow compensation. The anterior part of the RPE complex is shown by a red line, and the posterior part of the choroid is denoted by a green line. The yellow rectangles show the positions of retinal shadows. The black arrows indicate the new visible interfaces beneath retinal vessels. (Color online only.)

slice images of the $\mathrm{ONH}$ area at different depths. In both sequences, the first image is from the RPE, and the following images are sliced at $49 \mu \mathrm{m}$ intervals from it. The first en face images of both sequences, (a) and (b), in Fig. 3 are from the flattened RPE. By comparing these images, the effect of retinal shadow compensation can be seen clearly. If compensation is not used, retinal shadows cause artifacts that are erroneously segmented as choroidal vessels, as seen in the first slice of row (b). The en face slice images taken at a depth of $49 \mu \mathrm{m}$ below the RPE suggest that information can be obtained about the choriocapillaris structure, which contains many small blood vessels and pigment cells. ${ }^{4}$ However, due to the resolution limitations of OCT, a detailed investigation of blood vessels within the choriocapillaris is a very challenging task. Taking en face slice images at $98 \mu \mathrm{m}$ and $137 \mu \mathrm{m}$ below the RPE reduces the inverse intensity of the OCT signal around the optic disk area. This indicates that the thickness of choroid that contains vessels decreases toward the optic disk. The data also enable determining the depth position of vessels. The last images of sequences (a) and (b) in Fig. 3 show a 3-D reconstruction of choroidal vessels in the ONH. Volume (a) is obtained with shadow compensation and (b) without retinal shadow compensation.

In 3-D segmentation of choroidal vasculatures, a simple intensity threshold is applicable for the identification of choroidal vessels. In the absence of retinal shadow compensation, the calculated intensity threshold is distorted, and choroidal vessels with poor contrast are segmented out. This explains why the 3-D image (b) in Fig. 3 seems to have less vessels than image (a).

Although the presented method was developed primarily for SOCA, it also seems to work well for restoring OCT images in general. A comparison of the flattened cross-sectional image after steps ii and vi in Fig. 4 demonstrate the obvious improvement in image quality following shadow compensation. There seems to be choroidal structural information even beneath a large retinal vessel.

It has been shown previously that the SOCA method provides comparable results with conventionally used angiographic methods like FA and ICGA. ${ }^{3}$ In this paper, the SOCA method is improved so that artifacts caused by retinal vessels can be removed to enhance readability of obtained choroidal vessel structure images. The presented compensation method is based on the fact that there is still some real OCT signal beneath retinal vessels. The shape similarity of median A-scans with and without the effect of retinal vessels (see image after step $\mathrm{v}$ in Fig. 1) affirms that statement.
Despite good results obtained by the use of the shadow compensation algorithm, some small compensation errors still occur. One reason could be errors in segmenting retinal vessels, when shadow compensation is performed on A-scans that are not actually affected by retinal shadows. Another possible explanation is that the single offset vector $a(z)$ is used in the compensation procedure globally. Since attenuation beneath retinal vessels is not uniform, some small compensation errors may creep in, as shown in Fig. 3.

The retinal shadow compensation method is rather simple and not particularly extensive in terms of calculation time. A standard PC (CPU 2.4 GHz, 2.93-GB RAM) calculated the desired compensation values for our volume in about $10 \mathrm{~s}$. However, to work properly, the method requires rather exact RPE identification. Especially in the $\mathrm{ONH}$ area, segmentation errors beneath the optic disk may yield incorrect retinal shadow compensation values, affecting choroidal vessel segmentation. In Fig. 3, for example, the vessel structures visible beneath the optic disk cupping are not actual choroidal vessels, but artifacts produced by the shadows of retinal vessels. In fact, there is no choroid under the optic disk at all. Thus, segmentation of the optic disk area is a necessity.

This paper demonstrated shadow compensation on OCT images obtained using a conventional $840-\mathrm{nm}$ probing band. However, some longer wavelength OCT systems for ophthalmic imaging have been reported recently. ${ }^{2,4,5}$ One very interesting alternative is $1-\mu \mathrm{m}$ OCT, because it allows a deeper imaging depth than the 840-nm OCT, providing information even from the sclera. ${ }^{2}$ Although the optical properties of vessels and blood are wavelength dependent and the appearance of retinal shadows is different in 1- $\mu \mathrm{m}$ OCT than in $840 \mathrm{~nm}$ OCT, the fact remains that retinal shadows will still appear in both types of image. The applicability of the method presented here is independent of the wavelength used.

An automatic method for compensating for retinal shadows in OCT images is presented. By using this method, the readability of tomography images can be improved. Moreover, the presented compensation method also improves the efficiency of the choroidal vessel segmentation process and increases the visibility and contrast of choroidal vessels. This enables obtaining information about real choroidal structures located beneath retinal vessels.

\section{References}

1. S. Makita, Y. J. Hong, M. Yamanari, T. Yatagai, and Y. Yasuno, "Optical coherence angiography," Opt. Express 17, 7821-7840 (2006).

2. Y. Yasuno, Y. Hong, S. Makita, M. Yamanari, M. Akiba, M. Miura, and T. Yatagai, "In vivo high-contrast imaging of deep posterior eye by $1-\mu \mathrm{m}$ swept source optical coherence tomography and scattering optical coherence angiography," Opt. Express 15, 6121-6139 (2007).

3. Y. Hong, S. Makita, M. Yamanari, M. Miura, S. Kim, T. Yatagai, and Y. Yasuno "Three-dimensional visualization of choroidal vessels by using standard and ultra-high resolution scattering optical coherence angiography," Opt. Express 15, 7538-7550 (2007).

4. E. C. Lee, J. F. de Boer, M. Mujat, H. Lim, and S. H. Yun, "In vivo optical frequency domain imaging of human retina and choroid," Opt. Express 14, 4403-4411 (2006)

5. A. Unterhuber, B. Povazay, B. Hermann, H. Sattmann, A. ChavezPirson, and W. Drexler "In vivo retinal optical coherence tomography at $1040 \mathrm{~nm}$-enhanced penetration into the choroid," Opt. Express 13, 3252-3258 (2005). 